\title{
Proceeding
}

Supplementary Issue: Spring Conferences of Sports Science. 15th Convention and Workshop of the International Network of Sport and Health Science, 5-8 June 2019. University of Las Palmas de Gran Canaria, Las Palmas de Gran Canaria, Spain.

\section{Accuracy and reliability of a free mobile HRV application in measurement of heart rate variability}

\author{
JEFFREY PAGADUAN ${ }^{1}$, EDIN UŽIČANIN ${ }^{2,3}$, MELIKA MURATOVIĆ ${ }^{2,3}$, HARIS POJSKIĆ4,5 \\ ${ }^{1}$ College of Health and Medicine, School of Health Sciences, University of Tasmania, Tasmania, Australia \\ ${ }^{2}$ Center for Sports Excellence, Tuzla, Bosnia and Herzegovina \\ ${ }^{3}$ School of Physical Education and Sport, Tuzla University, Bosnia and Herzegovina \\ ${ }^{4}$ Department of Sports Science, Faculty of Social Sciences, Linnaeus University, Sweden \\ ${ }^{5}$ The Swedish Winter Sports Research Centre, Mid Sweden University, Östersund, Sweden
}

\begin{abstract}
Background: This study aimed to investigate the accuracy and reliability of a free mobile heart rate variability (HRV) application in measuring HRV. Methods: Twelve females and 12 males underwent five-minute simultaneous HRV recording from electrocardiogram (ECG) and chest strap connected to a free mobile HRV application (HRVapp) in a supine position. HRV data from ECG and HRVapp were used to examine accuracy and reliability via relative error and intraclass correlation coefficient respectively. Results: The natural log of the square root of the mean of the sum of the squares of differences between adjacent normal to normal intervals (InRMSSD) exhibited accuracy and high reliability in HRVapp. Conclusion: InRMSSD in HRVapp can serve as an alternative, low-cost technology for measurement of autonomic activity. Keywords: Heart rate variability; Mobile application; Autonomic health; Parasympathetic nervous system; Sympathetic nervous system; Autonomic activity.

\section{Cite this article as:}

Pagaduan, J., Užičanin, E., Muratović, M., \& Pojskić, H. (2019). Accuracy and reliability of a free mobile HRV application in measurement of heart rate variability. Journal of Human Sport and Exercise, 14(4proc), S578-S583. doi:https://doi.org/10.14198/jhse.2019.14.Proc4.15

Corresponding author. College of Health and Medicine, School of Health Sciences, University of Tasmania - Newnham Campus, Newnham Drive, Launceston, Tasmania 7250, Australia.

E-mail: jeffrey.pagaduan@utas.edu.au

Supplementary Issue: Spring Conferences of Sports Science. 15th Convention and Workshop of the International Network of Sport and Health Science, 5-8 June 2019. University of Las Palmas de Gran Canaria, Las Palmas de Gran Canaria, Spain. JOURNAL OF HUMAN SPORT \& EXERCISE ISSN 1988-5202

(c) Faculty of Education. University of Alicante doi:10.14198/jhse.2019.14.Proc4.15
\end{abstract}




\section{INTRODUCTION}

Heart rate variability $(\mathrm{HRV})$ refers to fluctuation in heartbeat intervals reflecting autonomic health (Bernston et al., 1997; Task Force, 1996). In athlete settings, practitioners utilize HRV in monitoring autonomic recovery and training load quantification (Janssen et al., 1993; Kylosov et al., 2009; Mal'tse et al., 2010; Plews, et al., 2012; Saboul et al., 2018; Schneider et al., 2018). The 'gold standard' for measuring HRV is through a laboratory electrocardiogram (ECG). However, the previous method is costly and require technical expertise. Recently, numerous low-cost HRV equipment are available. Among these is a smartphone with an HRV mobile application (HRVapp) (Boos et al., 2017; Flatt and Esco, 2013; Perrotta et al., 2017).

Equivocal findings in the validity of HRVapp exist. For example, Flatt and Esco (2013) found out that ultrashort term HRV using the log-transformed square root of the mean of the sum of the squares of differences between adjacent normal to normal intervals (InRMSSD) from an HRVapp is valid when compared with InRMSSD obtained from ECG. Conversely, Perrotta and colleagues (2017) discovered that an HRVapp presented poor validity when used for 14 -day monitoring.

The aforementioned HRVapp studies use RMSSD which is a time-based HRV index (Bernston et al., 1997; Task Force, 1996). Implications for autonomic health can be better understood using frequency derived HRV indices (Malliani et al., 1991; Malliani et al.,1994; Montano et al., 2009; Sassi et al., 2015; Schneider et al., 2018). However, there seems to be a void in literature examining the accuracy and reliability of frequencybased HRV from an HRVapp. Thus, the purpose of this study was to examine the accuracy and reliability of time and frequency HRV indices from an HRVapp.

\section{METHODS}

\section{Participants}

Twelve females (age: $20.3 \pm 0.97$ yrs.; height: $170 \pm 5.51 \mathrm{~cm}$; weight: $64.5 \pm 8.16 \mathrm{~kg}$; body mass index: 22.2 \pm 2.36 ) and 12 males (age: $21.3 \pm 1.48$ yrs.; height: $181 \pm 4.80 \mathrm{~cm}$; weight: $80.9 \pm 6.47 \mathrm{~kg}$; body mass index: $24.6 \pm 1.60$ ) volunteered to participate in the study. All participants were physical education students who have no known clinical disease. At the time of the study, participants reported at least $10 \mathrm{hrs}$ of physical activity per week. All participants were informed of the purpose, benefits, risks of the study, and right to withdraw at any time without explanation. Before commencing the study, participants signed a written informed consent. The study was performed in accordance with the Declaration of Helsinki for Human Experimentation and was approved by the local institutional ethical board.

\section{Procedures}

Experimentation occurred for a single session between $08.00-12.00$ hrs at the Tuzla University Exercise Science Laboratory. Upon arrival, participants were asked to a seated rest for 5 minutes. After, anthropometric data were collected. Body height was measured to the nearest $0.01 \mathrm{~m}$ from a portable stadiometer (Astra 27310; Gima, Italy). Body mass was identified using a portable scale (Tanita TBF-300, increments $0.1 \%$; Tanita, Tokyo, Japan). Anthropometric measurements were followed by placement an elastic strap with a heart transmitter (Polar H10, Polar Electro Oy, Kemple, Finland) around the upper thorax at the level of xiphoid process. A free HRVapp (Pulse Express Pro, Philippines) operating on an android smartphone (Xperia XA, Sony, UK) was utilized for HRV recording. Then, surface electrodes from a portable ECG (CONTEC8000G, Qinhuangdao Contec Medical Systems Co., Ltd, China) were attached using lead II configuration with a sampling rate of $1000 \mathrm{~Hz}$. After ECG electrode attachment, participants underwent a 7minute supine rest with eyes closed on an examination bed. Simultaneous HRV recording from HRVapp and 
ECG was administered in the last 5 minutes of supine rest. Prior to recording, participants were asked to breathe normally and remain still during the entire test.

Data displayed on ECG and HRVapp were encoded on a spreadsheet. For time-based HRV, the standard deviation of normal to normal intervals (SDNN), RMSSD, pNN50 (number of pairs of adjacent NN intervals differing by more than $50 \mathrm{~ms}$ in the entire recording divided by the total number of NN intervals) were utilized (Task Force 1996). Further, RMSSD from ECG and HRVapp were transformed to natural log to address nonuniformity of error (Flatt et al., 2013; Perotta et al., 2017). Low frequency (LF: $0.04-0.15$ Hz; ms2), high frequency (HF: $0.15-0.4 \mathrm{~Hz}$; ms2), and LF/HF ratio were examined in frequency HRV domains. Normalized low frequency (LFnu $=L F / L F+H F * 100)$ and normalized high frequency (HFnu $=H F / L F+H F * 100)$ were also computed for comparison. Researchers suggested that LFnu and HFnu are reliable markers for quantifying autonomic function (Burr, 2007; Malliani et al., 1991; Malliani et al., 1994; Montano et al., 2009; Reyes del Paso et al., 2015).

\section{Data analysis}

Data are expressed as mean \pm standard deviation (SD). Relative error (\%RE) was used to identify the accuracy the HRV parameters in HRVapp (Abbiss et al., 2009). Equipment reliability of HRVapp was determined utilizing intraclass correlation coefficient (ICC) from the reliability spreadsheet by Hopkins (Atkinson and Nevill, 1998; Koo and Lee, 2016; Hopkins, 2000; Weir, 2005).

\section{RESULTS}

Table 1 displays the HRV parameters in ECG and HRVapp. Results showed that HRVapp SDNN posted $\%$ RE $=-6.326 \mathrm{~ms}$ and \%RE SDNN ICC $=0.941 . \%$ RE for HRVapp RMSSD was -6.326 ms with RMSSD ICC of 0.931 . HRVapp InRMSSD \%RE $=-0.667 \mathrm{~ms}$ and InRMSSD ICC $=0.941 . \%$ RE PNN50 for HRVapp was 34.38 while PNN50 ICC $=0.760$. For HRV frequency parameters, \%RE of HRVapp LF $=-139.5 \mathrm{~ms} 2$ and LF ICC $=.778$. HRVapp LFnu \%RE $=-3.074$ with LFnu ICC of 0.555 . HRVapp \%RE HF was -180.9 ms2 and HF ICC $=0.632 . \%$ RE HFnu of HRVapp $=12.69$ while HFnu ICC $=0.554$. Lastly, HRVapp \%RE LF/HF $=-589.8$ with $\mathrm{LF} / \mathrm{HF}$ ICC $=0.534$.

Table 1. HRV Indices in ECG and HRVapp (mean \pm SD)

\begin{tabular}{lccccc}
\hline & & ECG & HRVapp & \% RE & ICC \\
\hline SDNN & $(n=24)$ & $60.16 \pm 25.14$ & $63.45 \pm 26.60$ & -6.326 & 0.941 \\
RMSSD & $(n=24)$ & $56.32 \pm 32.95$ & $59.93 \pm 36.41$ & -6.326 & 0.931 \\
InRMSSD & $(n=24)$ & $3.854 \pm 0.655$ & $3.886 \pm 0.732$ & -0.667 & 0.941 \\
PNN50 & $(n=22)$ & $12.64 \pm 7.146$ & $27.79 \pm 15.69$ & 34.38 & 0.760 \\
LF & $(n=24)$ & $700.8 \pm 681.8$ & $1447 \pm 1270$ & -139.5 & 0.778 \\
LFnu & $(n=24)$ & $54.70 \pm 17.69$ & $54.24 \pm 19.59$ & -3.074 & 0.555 \\
HF & $(n=24)$ & $661.1 \pm 839.7$ & $1411 \pm 1454$ & -180.9 & 0.632 \\
HFnu & $(n=24)$ & $45.30 \pm 17.69$ & $45.76 \pm 19.59$ & -12.69 & 0.554 \\
LF/HF & $(n=24)$ & $1.632 \pm 1.313$ & $5.674 \pm 5.544$ & -589.8 & 0.534 \\
\hline ECG
\end{tabular}

ECG - electrocardiogram; HRVapp - Pulse Express Pro mobile application; \%RE - relative error; ICC - intraclass correlation coefficient; SDNN - standard deviation of normal to normal intervals (ms); RMSSD - square root of the mean of the sum of the sum of the squares of differences between adjacent normal to normal intervals (ms); InRMSSD - natural log of RMSSD (ms); PNN50 - number of pairs of adjacent normal to normal intervals different by more than $50 \mathrm{~ms}$ in the entire recording divided by the total number of normal to normal intervals (\%); LF - low frequency ms2; LFnu - normalized low frequency; HF - high frequency $m s^{2} ; H F n u$ - normalized high frequency; LF/HF - low frequency to high frequency ratio. 


\section{DISCUSSION}

The aim of this study was to establish the accuracy of a free HRVappn measuring HRV parameters when compared to the ECG criterion. Accuracy was assessed using \%RE interpreted from the following criteria: < $0.50 \%$ - accurate; 1.50 - $2.50 \%$ - moderately accurate; > $2.50 \%$ - inaccurate (Abbiss et al., 2009). Results showed that only InRMSSD was an accurate HRV measure. This finding coincides with the results posted by previous researchers (Boos et al., 2016; Flatt et al., 2013). Further, other HRV parameters such as SDNN, RMSSD, LF, LFnu, HF, HFnu, LF/HF were found to be inaccurate. The observed inaccuracies from the HRVapp can be attributed to equipment variability from signal processing and artefact correction.

Another objective of this study was to examine the reliability of HRV indices from HRVapp. ICC was utilized for reliability which is interpreted as: ICC > 0.90 - 'excellent'; 0.80 - 0.90 - 'good'; < 0.80 - 'questionable' (Vincent, 2005). Findings demonstrated that ICC for SDNN, RMSSD, InRMSSD were 'excellent'. The results were in line with previous research (Flatt et al., 2013; Perotta et al., 2017). Further, ICC of PNN50 and LF are relatively 'good'. Reliability was seen to be 'poor' in LFnu, HF, HFnu, and LF/HF.

Limitations in this current study should be noted. Firstly, raw data from both ECG and HRVapp were not available. Extraction of raw data from both equipment may allow standardization of artefact correction and possibly increase the accuracy and reliability of HRV indices from HRVapp (Perotta et al., 2017). Also, computation of HRV in HRVapp did not undergo artefact correction. This may have contributed to large 'noise' in HRV frequency parameters. Additionally, InRMSSD was not displayed in HRVapp. Inclusion of InRMSSD display feature after HRV recording in HRVapp can increase the utility of HRVapp. Lastly, equipment functionality and other reliability measures (e.g. intra/inter day) were not analysed in this study (Weir, 2005; Pyne et al, 2004). Carrying out such analyses may increase practitioner confidence in using the HRVapp. Thus, future studies addressing the limitations of this study should be warranted.

In conclusion, InRMSSD is an accurate and highly reliable index in measuring autonomic modulation in HRVapp. On the other hand, the high reliability demonstrated by SDNN and RMSSD in HRVapp suggest that these measures can be utilized in monitoring autonomic activity over time.

\section{PRACTICAL IMPLICATIONS}

Practitioners can use the HRVapp as a low-cost alternative to measure autonomic health via InRMSSD.

\section{CONFLICT OF INTEREST}

The authors have no conflict of interest to declare. No funding was used for this study.

\section{ACKNOWLEDGEMENT}

The authors are thankful to the participants who volunteered for this study.

\section{REFERENCES}

Abbiss, C.R., Quod, M.J., Levin, G., Martin, D.T., \& Laursen, PB. (2009). Accuracy of the Velotron ergometer and SRM power meter. International Journal of Sports Medicine, 30, 107-112. https://doi.org/10.1055/s-0028-1103285 
Atkinson, G., \& Nevill, A.M. (1998). Statistical methods for assessing measurement error (reliability) in variables relevant to sports medicine. Sports Medicine, 26(4), 217-238. https://doi.org/10.2165/00007256-199826040-00002

Bernston, G.G., Bigger Jr., J.T., Eckberg, D.L., Grossman, P., Kaufmann, P.G., Malik, M., ... van Der Molen, M.W. (1997). Heart rate variability: origins, methods, and interpretive caveats. Psychophysiology, 1934(6), 623-648. https://doi.org/10.1111/j.1469-8986.1997.tb02140.x

Boos, C.J., Bakker-Dyos, J., Watchorn, J., Woods, D.R., O'Hara, J.P., Macconnachie, L., ... Mellor, A. (2017). A comparison of two methods of heart rate variability assessment at high altitude. Clinical Physiology and Functional Imaging, 3(6), 582- 587. https://www.doi.org/10.1111/cpf.12334

Burr, R.L. (2007). Interpretation of normalized spectral heart rate variability indices in sleep research: a critical review. Sleep, 30(7), 913-919. https://doi.org/10.1093/sleep/30.7.913

Flatt, A.A., \& Esco, M.R. (2012). Validity of the ithlete smart phone application for determining ultra-shortterm heart rate variability. Journal of Human Kinetics, 39, 85-92. https://doi.org/10.2478/hukin2013$\underline{0071}$

Janssen, M.J., de Bie, J., Swenne, C.A., \& Oudhof, J. (1993). Supine and standing sympathovagalbalance in athletes and controls. European Journal of Applied Physiology, 67(2), 164167. https://doi.org/10.1007/bf00376661

Koo, T.K., \& Li, M.Y. (2016). A guideline of selecting and reporting intraclass correlation coefficients for reliability research. Journal of Chiropractic Medicine, 15(2), 155-163. https://doi.org/1016/i.jcm.2016.02.012

Hopkins, W.G. (2000). Measures of reliability in sports medicine and science. Sports Medicine, 30(1), 115.

Kylosov, A.A., Mel'nikov, A.A., Mal'tsev, A., Vikulov, A.D., \& Borisova, O.L. (2009). [Changes in inflammatory activity, heart rate variability, and biochemical indices in young athletes during the annual training cycle]. Fiziologiia Cheloveka, 35(4), 82-96. https://doi.org/10.1134/s0362119709040124

Malliani, A., Lombardi, F., \& Pagani, M. (1994). Power spectrum analysis of heart rate variability: a tool to explore neural regulatory mechanisms. British Heart Journal, 84, 482-492. https://doi.org/10.1136/hrt.71.1.1

Malliani, A., Pagani, M., Lombardi, F., \& Cerutti, S. (1991). Cardiovascular neural regulation explored in the frequency domain. Circulation, 84, 482-492. https://doi.org/10.1161/01.cir.84.2.482

Mal'tsev, A.Y., Mel'nikov, A.A., Vikulov, A.D., \& Gromova, K.S. (2010). Central hemodynamic and heart rate variability parameters in athletes during different trainng programs. Human Physiology, 36(1), 96-101. https://doi.org/10.1134/S0362119710010135

Montano, N., Porta, A., Cogliati, C., Costantino, G., Tobaldini, E., Casali, K.R., lellamo, F. (2009). Heart rate variability explored in the frequency domain: a tool to investigate the link between heart and behavior. Neuroscience and Biobehavioral Reviews, 33(2), 71-80. https://doi.org/10.1016/i.neubiorev.2008.07.006

Perrotta, A.S., Jeklin, A., Hives, B.A., Meanwell, L.E., Warburton, D.E. (2017). Validity of the elite HRV smart phone application for examining heart rate variability in a field based setting. Journal of Strength and Conditioning Research, 31(8) 2296-2302. https://doi.org/10.1519/jsc.0000000000001841

Plews, D.J., Laursen, P.B., Kilding, A.E., \& Buchheit, M. (2012). Heart rate variability in elite triathletes, is variation in variability the key to effective training? A case comparison. European Journal of Applied Physiology, 112(11), 3729-3741. https://doi.org/10.1007/s00421-012-2354-4 
Pyne, D., Trewin, C., \& Hopkins, W. 2004. Progression and variability of competitive performance of olympic swimmers. Journal of Sports Science, 22(7): 613-620. https://doi.org/10.1080/02640410310001655822

Reyes del Paso, G.A., Langewitz, W., Mulder, L.J., van Roon, A., \& Duschek, S. (2013). The utility of low frequency heart rate variability as an index of sympathetic cardiac tone: a review with emphasis on a reanalysis of previous studies. Psychophysiology, 50(5), 477-487. https://doi.org/10.1111/psyp.12027

Saboul, D., Balducci, P., Millet, G., Pialoux, V., \& Hautier, C. (2018). A pilot study on quantification of training load: the use of HRV in training practice. European Journal of Sports Science, 16(2), 172181. https://doi.org/10.1080/17461391.2015.1004373

Sassi, R,, Cerutti, S., Lombardi, F., Malik, M., Huikuri, H.V., Peng, C.K., ... Yamamoto, Y. (2015). Advances in heart rate variability analysis: joint position statement by the e-Cardiology ESC Working Group and the European Heart Rhythm Association co-endorsed by the Asia Pacific Heart Rhythm Society. Europace, 17(9),1341-1353. https://doi.org/10.1093/europace/euv015

Schneider, C., Hanakam, F., Wiewelhove, T., Döweling, A., Kellmann, M., Meyer, T., ... Ferrauti, A. (2018). Heart rate monitoring in team sports - a conceptual framework for contextualizing heart rate measures for training and recovery prescription. Frontiers in Physiology, 9, 639. https://doi.org/10.3389/fphys.2018.00639

Task Force of the European Society of Cardiology and the North American Society of Pacing and Electrophysiology (Task Force). (1996). Heart rate variability: standards of measurement, physiological interpretation and clinical use. Circulation, 93(5), 1043-1065. https://doi.org/10.1111/j.1542-474x.1996.tb00275.x

Vincent, W. (2005). Statistics in Kinesiology (4th ed). Champaign, IL: Human Kinetics.

Weir, J.P. (2005). Quantifying test-rest reliability using the intraclass correlation coefficient and the SEM. Journal of Strength and Conditioning Research, 19(1), 231-240. https://doi.org/10.1519/00124278$\underline{200502000-00038}$

\section{(9)}

This work is licensed under a Attribution-NonCommercial-NoDerivatives 4.0 International (CC BY-NC-ND 4.0). 